\title{
The Interprocedural Coincidence Theorem
}

\author{
Jens Knoop * Bernhard Steffen ${ }^{\dagger}$
}

\begin{abstract}
We present an interprocedural generalization of the well-known (intraprocedural) Coincidence Theorem of Kam and Ullman, which provides a sufficient condition for the equivalence of the meet over all paths (MOP) solution and the maximal fixed point (MFP) solution to a data flow analysis problem. This generalization covers arbitrary imperative programs with recursive procedures, global and local variables, and formal value parameters. In the absence of procedures, it reduces to the classical intraprocednral version. In particular, our stack-based approach generalizes the coincidence theorems of Barth and Sharir/Pnueli for the same setup, which do not properly deal with local variables of recursive procedures.
\end{abstract}

\section{Motivation}

Data flow analysis is a classical method for the static analysis of programs that supports the generation of efficient object code by "optimizing" compilers (cf. [He, MJ]). For imperative languages, it provides information about the program states that may occur at some given program points during execution.

Theoretically well-founded are data flow analyses that are based on abstract interpretation (cf. [CC1]). The point of this approach is to replace the "full" semantics by a simpler more abstract version, which is tailored to deal with a specific problem. Usually, the abstract semantics is specified by a local semantic functional, which gives abstract meaning to every program statement in terms of a transformation function from a lattice $\mathcal{C}$ into itself. The elements of $\mathcal{C}$ express the data flow information of interest. The (global) abstract semantics then results from one of the following two globalization strategies; the "operational" meet over all paths (MOP) strategy, and the "denotational" maximal fixed point (MFP) strategy ${ }^{1}$ in the sense of Kam and Ullman [KU] ${ }^{2}$.

The $M O P$-strategy directly mimics possible program executions: it "meets" (intersects) all information corresponding to program paths reaching the program point under consideration. This specifies the optimal result of a globalization but is in general not effective.

The MFP-strategy iteratively approximates the greatest solution of a system of equations that express consistency between pre-conditions and post-conditions that are given in terms of data flow information: the pre-condition of a statement must be implied by each of the post-conditions of the predecessors, and the post-condition must be implied by the result of transforming the pre-condition according to the (abstract) meaning of the statement. In general, this leads to a suboptimal but algorithmic description.

The (intraprocedural) Coincidence Theorem of [KU] states the coincidence of the MOP-solution and the $M F P$-solution in the case of distributive (local) semantic functionals (see Section 2). In this paper, we present an interprocedural generalization of this theorem (cf. Interprocedural Coincidence

\footnotetext{
*Institut für Informatik und Praktische Mathematik, Christian-Albrechts-Universität, Preußerstr. 1-9, D-2300 Kiel 1-Part of the work was done, while the author was supported by the Deutsche Forschungsgemeinschaft grant La 426/9-2.

'Lehrstuhl für Informatik II, Rheinisch-Westfalische Technische Hochschule Aachen, Ahornstr. 55, D-5100 Aachen.

'These are the strategies that lead to the $M O P$-solution and $M F P$-solution, respectively.

${ }^{2}$ The operational and denotational flavour of these two strategies becomes particularly apparent in the interprocedural setting.
} 
Theorem 5.3), which covers arbitrary imperative programs with recursive procedures, global and local variables, and formal value parameters. In the absence of procedures, it reduces to the classical intraprocedural version of Kam and Ullman. The point of our generalization is the introduction of stacks of lattice elements as data flow information, which is necessary to properly deal with local variables of recursive procedures. These stacks directly mimic the run-time stacks used by run-time systems for maintaining the activation records of different procedure incarnations. Whereas the operational $(M O P)$ strategy exhaustively makes use of the stack structure, it turns out that the denotational (MFP) strategy only needs stacks of length at most two (Remark 4.7).

\section{Related Work}

Semantically based reasoning about interprocedural data flow analysis was first considered by Cousot/ Cousot [CC2], Rosen [Ro], Jones and Muchnick [JM], and more recently by Bourdoncle [Bo].

Rosen [Ro] proved the correctness of an interprocedural data flow analysis algorithm computing a maximal fixed point solution that provides information whether a variable is used, modified or preserved. Thus his algorithm is tailored to specific problems, and it is not clear how to generalize his approach to arbitrary abstract interpretations. Such a general situation was investigated in [CC2] and [JM], leading to highly technical definitions that are difficult to apply. Moreover, as in [Ro], these two papers only address the correctness aspect but no optimality. This holds also for the approach of [Bo], which considers correctness of data flow analyses with respect to the collecting semantics of a program.

Both correctness and optimality were considered by Barth [Ba1, Ba2] and Sharir/Pnueli [SP], who independently proposed an interprocedural version of the Coincidence Theorem of Kam and Ullman. However, their approaches do not properly deal with local variables of recursive procedures, which would require to store information about the local variables when treating a recursive procedure call.

\section{Structure of the Paper}

After sketching the intraprocedural setting in Section 2, we recall the formal framework for interprocedural data flow analysis in Section 3, and define two versions of abstract semantics for programs with procedures in Section 4: an "operational" one caused by the interprocedural meet over all paths strategy and a "denotational" one caused by the interprocedural maximal fixed point strategy. Subsequently, Section 5 presents the main results, Section 6 sketches some applications, and Section 7 contains our conclusions. Finally, the Appendix provides the detailed data flow analysis algorithms.

\section{The Intraprocedural Setting}

In this section we summarize the intraprocedural setting for data flow analysis, which is characterized by a separate and independent investigation of the procedures of a program. Here it is common to represent procedures as directed flow graphs $G=(N, E, \mathbf{s}, \mathbf{e})$ with node set $N$ and edge set $E .^{3}$ Nodes $n \in N$ represent the statements and edges $(n, m) \in E$ the nondeterministic branching structure of the corresponding procedure. $\operatorname{pred}_{G}(n)==_{d f}\{m \mid(m, n) \in E\}$ and $\operatorname{succ}_{G}(n)=_{d f}\{m\}$ $(n, m) \in E\}$ denote the set of all immediate predecessors and successors of a node $n$, respectively. $\mathrm{s}$ and $\mathrm{e}$ denote the unique start node and end node of $G$, which are assumed to possess no predecessors and successors, respectively. A finite path in $G$ is a sequence $\left(n_{1}, \ldots, n_{q}\right)$ of nodes such that $\left(n_{j}, n_{j+1}\right) \in E$ for $j \in\{1, . . q-1\}$. P $[m, n]$ denotes the set of all finite paths from $m$ to $n$, and $\mathbf{P}[m, n)$ the set of all finite paths from $m$ to a predecessor of $n$. Moreover, $\operatorname{lgth}(p)$ denotes the number of node occurrences in $p$, and $\varepsilon$ the unique path of length 0 . Finally, we assume that every node $n \in N$ lies on a path from $s$ to $e$.

\footnotetext{
${ }^{3}$ The construction of flow graphs is described in [All].
} 
Given a complete semi-lattice $(\mathcal{C}, \sqcap, \sqsubseteq, \perp, T)$, whose elements are intended to express the relevant data flow information, the local abstract semantics of a flow graph $G$ is given by a semantic functional

$$
\llbracket \rrbracket: N \rightarrow(\mathcal{C} \rightarrow \mathcal{C})
$$

which gives meaning to every node $n \in N$ in terms of a transformation on $\mathcal{C}$. For simplicity, it is assumed that $\mathbf{s}$ and $\mathbf{e}$ are associated with the identity on $\mathcal{C}$.

The local abstract semantics 【 \ can easily be extended to cover finite paths as well. For every path $p=\left(n_{1}, \ldots, n_{q}\right) \in \mathbf{P}[m, n]$, we define:

$$
\llbracket p \rrbracket= \begin{cases}i d_{\mathcal{C}} & \text { if } p \equiv \varepsilon \\ \llbracket\left(n_{2}, \ldots, n_{q}\right) \rrbracket \circ \llbracket n_{1} \rrbracket & \text { otherwise }\end{cases}
$$

The (global) abstract semantics then results from one of the following two globalization strategies: the "operational" meet over all paths (MOP) strategy, and the "denotational" maximal fixed point (MFP) strategy in the sense of Kam and Ullman [KU].

The $M O P$-strategy directly mimics possible program executions: it "meets" (intersects) all informations, which belong to a program path reaching the program point under consideration:

The MOP-Solution: $\forall n \in N \forall c_{0} \in \mathcal{C} . M O P_{c_{0}}(n)=\prod\left\{\left\lfloor p \rrbracket\left(c_{0}\right) \mid p \in \mathbf{P}[\mathbf{s}, n)\right\}\right.$

This directly reflects our desires but is in general not effective,

The MFP-strategy iteratively approximates the greatest solution of a system of equations which specifies the consistency between pre-conditions and post-conditions that are expressed in terms of $\mathcal{C}:$

\section{Equation System 2.1}

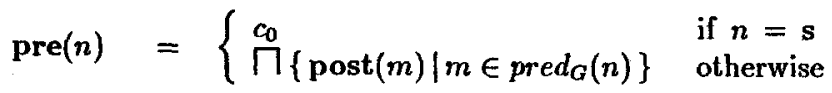

$$
\begin{aligned}
& \operatorname{post}(n)=\lfloor n \rrbracket(\operatorname{pre}(n))
\end{aligned}
$$

Denoting the greatest solution of Equation System 2.1 with respect to the start information $c_{0} \in \mathcal{C}$ by pre $_{c_{0}}$ and post $c_{c_{0}}$, the solution of the MFP-strategy is defined by:

The MFP-Solution: $\forall n \in N \forall c_{0} \in \mathcal{C} . M_{c_{0}}(n)=\operatorname{pre}_{c_{0}}(n)$

In general, this leads to a suboptimal but algorithmic description.

Thus we have two global notions of semantics here, an operational one, which precisely mimics our intention, and a denotational one, which has an algorithmic character. In fact, we consider the MOPstrategy as a mean for the direct specification of data flow analysis problems, and the $M F P$-strategy as an algorithmic realization of such problems ${ }^{4}$. This view rises the question of correctness (safety) or even completeness (optimality) of such algorithms. For the elegant answer to these questions we need two further notions: given a complete semi-lattice $(\mathcal{C}, \Pi, \subseteq, \perp, T)$, a function $f: \mathcal{C} \rightarrow \mathcal{C}$ is called

- monotonic iff $\forall c, c^{\prime} \in \mathcal{C} . c \sqsubseteq c^{\prime}$ implies $f(c) \sqsubseteq f\left(c^{\prime}\right)$

- distributive iff $\forall C^{\prime} \subseteq \mathcal{C}$. $f\left(\Pi C^{\prime}\right)=\Pi\left\{f(c) \mid c \in C^{\prime}\right\}$

It is well-known that distributivity is a stronger requirement than monotonicity in the following sense:

\footnotetext{
${ }^{4}$ Explicit algorithms are presented in Appendix A.
} 
Lemma 2.2

A function $f: \mathcal{C} \rightarrow \mathcal{C}$ is monotonic iff $\forall C^{\prime} \subseteq \mathcal{C} . f\left(\Pi C^{\prime}\right) \sqsubseteq \Pi\left\{f(c) \mid c \in C^{\prime}\right\}$

As in this lemma, $\mathcal{C}$ will always denote a complete semi-lattice. We have (cf. [KU]):

Theorem 2.3 (Safety Theorem)

Given a flow graph $G=(N, E, \mathrm{~s}, \mathrm{e})$, the $M F P$-solution is a correct (or safe) approximation of the $M O P$-solution, i.e. $\forall n \in N \forall c_{0} \in \mathcal{C} . M F P_{c_{0}}(n) \sqsubseteq M O P_{c_{0}}(n)$, if all the semantic functions 【n】 are monotonic.

Distributivity of the semantic functions yields completeness (optimality). This follows from the well-known intraprocedural Coincidence Theorem 2.4 of [KU]:

Theorem 2.4 (Coincidence Theorem)

Given a flow graph $G=(N, E, \mathrm{~s}, \mathrm{e})$, the $M F P$-solution is complete (or optimal) for the MOP. solution, i.e. $\forall n \in N \forall c_{0} \in \mathcal{C} . M O P_{c_{0}}(n)=M F P_{c_{0}}(n)$, if all the semantic functions 【n】are distributive.

\section{Interprocedural Notions}

In the interprocedural setting we represent programs $\Pi$ as systems $\left(\pi_{0}, \pi_{1}, ., \pi_{k}\right)$ of (recursive) procedure definitions, where every $\pi \in \Pi$ has a list of formal value parameters and a list of local variables. $\pi_{0}$ is assumed to denote the main program and therefore cannot be called. $\pi_{1}$ up to $\pi_{k}$ are the procedure declarations of $\Pi$. For simplicity we assume that there is no (static) procedure nesting except that $\pi_{0}$ encloses $\pi_{1}$ up to $\pi_{k} .{ }^{5}$ Thus, the variables of the main program are global variables of the procedures, and can be accessed by them.

The denotational (IMFP) approach and the operational (IMOP) approach require different representations of programs II: flow graph systems and interprocedural flow graphs.

\section{Flow Graph Systems}

The denotational approach works on systems $S=\left(G_{0}, G_{1}, ., G_{k}\right)$ of flow graphs with disjoint sets of nodes $N_{i}$ and edges $E_{i}$, in which every procedure $\pi$ of $\Pi$ (including the main program $\pi_{0}$ ) is represented as a directed flow graph $G=(N, E, \mathrm{~s}, \mathrm{e})$ in the sense of Section $2 . \mathrm{N}^{S}={ }_{d f} \bigcup\left\{N_{i} \mid i \in\right.$ $\{0, ., k\}\}$ denotes the set of all nodes of $S, \mathbf{E}^{S}=d j \bigcup\left\{E_{i} \mid i \in\{0, ., k\}\right\}$ the set of all edges of $S$, and $\mathbf{N}_{C}^{S} \subseteq \mathbf{N}^{S}$ the set of all nodes representing procedure calls. Finally, we need the following functions, where $\mathcal{P}$ denotes the power set operator:

- $f g: \mathrm{N}^{S} \rightarrow S$ with $f g(n)=_{\mathrm{d} f} G_{i}$ iff $n \in N_{i}$,

- callee $: \mathbf{N}_{C}^{S} \rightarrow S$ with callee $(n)={ }_{d f} G_{i}$ iff $n$ repesents a procedure call of $G_{i}$,

- caller $: S \rightarrow \mathcal{P}\left(\mathbf{N}_{C}^{S}\right)$ with caller $\left(G_{i}\right)={ }_{d f}\left\{n \mid\right.$ callee $\left.(n)=G_{i}\right\}$,

- start $: S \rightarrow\left\{\mathrm{s}_{0}, ., \mathrm{s}_{k}\right\}$ with $\operatorname{start}\left(G_{i}\right)={ }_{d f} \mathrm{~s}_{i}$ for all $i \in\{0, ., k\}$ and

- end $: S \rightarrow\left\{\mathbf{e}_{0}, ., \mathbf{e}_{k}\right\}$ with $\operatorname{end}\left(G_{i}\right)={ }_{d j} \mathbf{e}_{i}$ for all $i \in\{0, ., k\}$.

Intuitively, $f g$ maps every node of a flow graph system to its corresponding low graph, callee every call node to the called procedure, caller every procedure to its set of call nodes, and start and end every procedure to its start node and its end node, respectively.

An illustrative flow graph system is given in Figure 1.

\footnotetext{
${ }^{5}$ Integrating static procedure nesting is straightforward.
} 

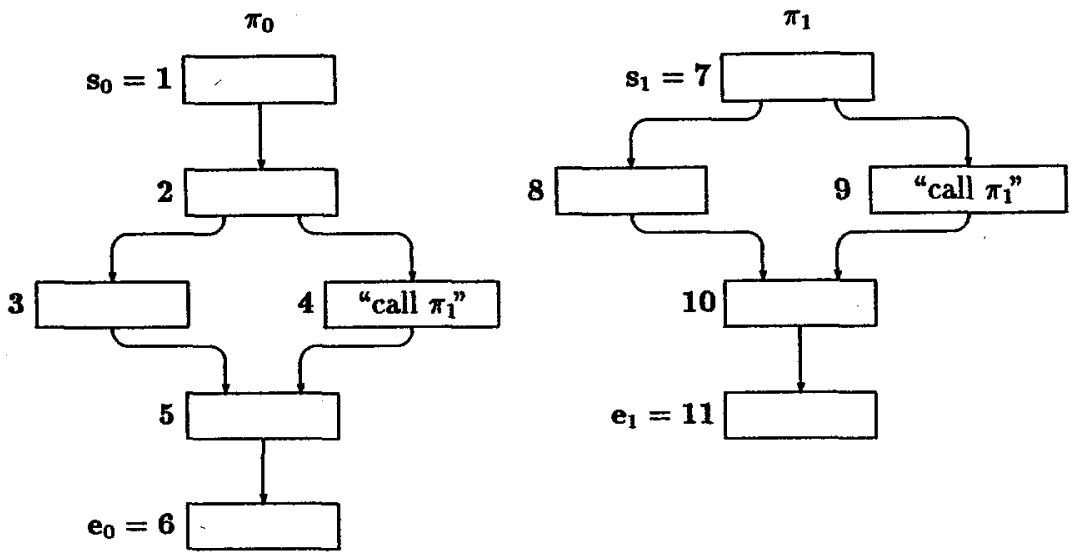

Figure 1: The Flow Graph System

\section{Interprocedural Flow Graphs}

The operational approach requires an explicit representation of the interprocedural control flow caused by procedure calls. This is achieved by combining the flow graphs of $S$ to an interprocedural flow graph $G^{*}=\left(N^{*}, E^{*}, \mathbf{s}^{*}, \mathrm{e}^{*}\right)$, where $\mathrm{s}^{*}$ is given by $\mathrm{s}_{0}$ and $\mathrm{e}^{*}$ by $\mathbf{e}_{0}$ (cf. [My, SP]). In detail, $G^{*}$ results from $S$ by applying the following two step procedure to every node $n$ of $\mathrm{N}_{C}^{S}$ :

Algorithm 3.1 Let $S$ be a flow graph system, and $n \in \mathbf{N}_{C}^{S}$. Then

1. Replace $n$ by two new nodes, the call node $n_{C}$ and the return node $n_{R}$ such that $n_{C}$ has the same set of predecessors as $n$ but no successors and $n_{R}$ has the same set of successors as $n$ but no predecessors ${ }^{6}$.

2. Draw an edge from $n_{C}$ to start(callee(n)) and from end(callee(n)) to $n_{R}$.

$N_{C}^{*}$ and $N_{R}^{*}$ denote the set of all call nodes and return nodes in $N^{*}$, respectively, and pred ${ }^{*}(n)={ }_{d f}$ $\left\{m \mid(m, n) \in E^{*}\right\}$ the set of all immediate interprocedural predecessors of $n$. In the following, we will identify the set $\mathrm{N}^{S}$ of nodes of $S$ with the set $N^{*} \backslash N_{R}^{*}$ of nodes of $G^{*}$ to get an interpretation independent notion of program point. 1 .

Figure 2 shows the interprocedural flow graph that corresponds to the flow graph system of Figure

\section{Interprocedural Paths}

The notion of finite path as introduced in Section 2 naturally applies to interprocedural flow graphs as well. However, due to the special nature of procedure calls not every finite path $p \in \mathbf{P}[m, n]$ represents a valid execution. For example in Figure 2 the path $\left(2,4_{C}, 7,8,10,11,4_{R}\right)$ is possible, while the path $\left(2,4_{C}, 7,8,10,11,9_{R}\right)$ is not. This led to the following definition of interprocedural (valid) paths [SP]:

\section{Definition 3.2 (Interprocedural Path)}

1. Let $p \in \mathbf{P}[m, n]$. Then $p$ is an interprocedural path iff the tuple $\left(m_{1}, ., m_{r}\right)$, which results from $p$ by deleting all nodes in $N^{*} \backslash\left(N_{C}^{*} \cup N_{R}^{*}\right)$, is well-formed in the following sense:

- if there is no return node in $\left(m_{1}, . ., m_{r}\right)$, then $\left(m_{1}, ., m_{r}\right)$ is well-formed,

\footnotetext{
${ }^{6} n_{C}$ is called to match $n_{R}$ and vice versa.
} 


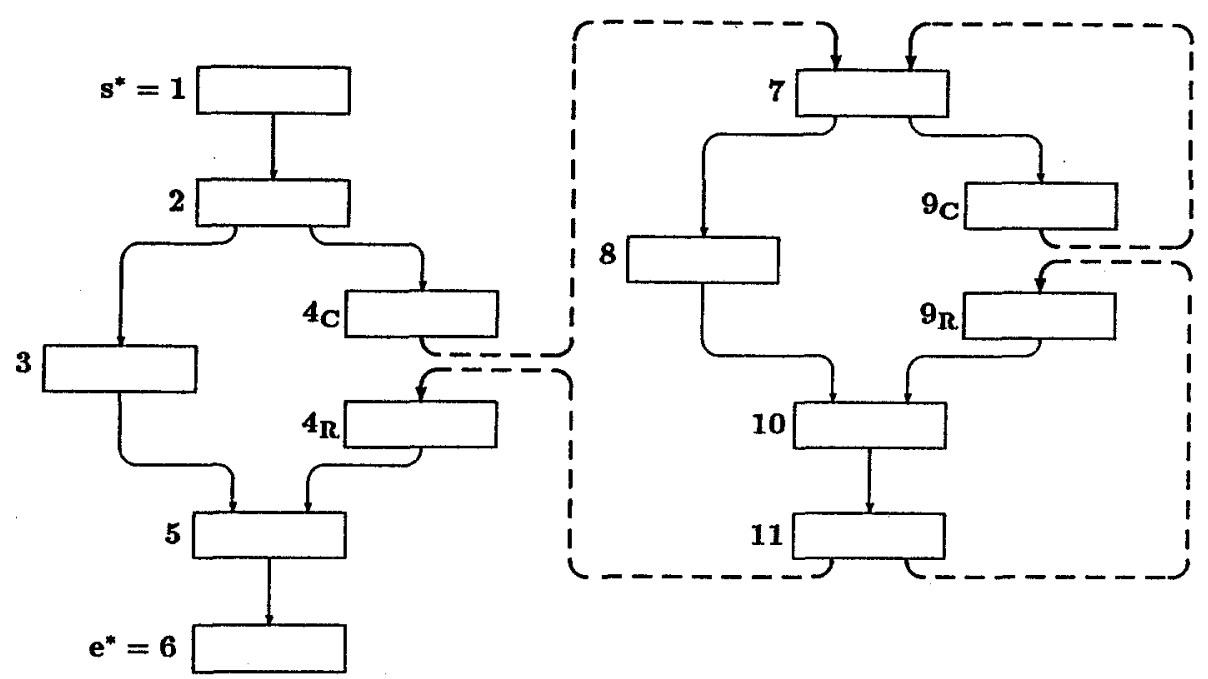

Figure 2: The Interprocedural Flow Graph

- otherwise, let $j$ be the smallest index in $\{1, ., r\}$ such that $m_{j}$ is a return node. Then $\left(m_{1}, ., m_{r}\right)$ is well-formed iff $j>1$ and $m_{j-1}$ is a call node matching $m_{j}$, and the remaining sequence after deleting $m_{j-1}$ and $m_{j}$ is well-formed too.

2. A call node and a return node of $p$ are said to correspond to each other, if they are eliminated simultaneously in the procedure above.

3. IP $[m, n]$ denotes the set of all interprocedural paths from $m$ to $n$, and $\operatorname{IP}[m, n)$ the set of all interprocedural paths from $m$ to a predecessor of $n$.

\section{Complete Interprocedural Paths}

In order to determine the semantics of procedure calls, we need to deal with complete interprocedural paths $p$ from $\operatorname{start}(f g(n))$ to $n$, which are characterized by the fact that all procedure calls in $p$ have been completed by a subsequent return. This guarantees that the occurrences of $\operatorname{start}(f g(n))$ and $n$ belong to the same procedure incarnation.

\section{Definition 3.3 (Complete Interprocedural Path)}

1. An interprocedural path $p=\left(n_{1}, \ldots, n_{k}\right) \in \mathbf{I P}[\operatorname{start}(f g(n)), n]$ is called complete if it possesses equally many occurrences of procedure call and return nodes:

$$
\left|\left\{i \mid n_{i} \in N_{C}^{*}\right\}\right|=\left|\left\{i \mid n_{i} \in N_{R}^{*}\right\}\right|
$$

2. CIP $[\operatorname{start}(f g(n)), n]$ and $\operatorname{CIP}[\operatorname{start}(f g(n)), n)$ denote the set of all complete interprocedural paths from start $(f g(n))$ to $n$, and from $\operatorname{start}(f g(n))$ to a predecessor of $n$, respectively.

That this actually realizes our intention is a consequence of the following property of interprocedural paths:

Lemma 3.4 Let $p=\left(n_{1}, \ldots, n_{k}\right) \in \operatorname{IP}[m, n]$ be an interprocedural path and $\left(n_{i}, n_{j}\right)$ and $\left(n_{i^{\prime}}, n_{j^{\prime}}\right)$ two of its pairs of corresponding call and return nodes. Then the integer intervals $[i: j]$ and $\left[i^{\prime}: j^{\prime}\right]$ are either disjoint or one is included in the other. 


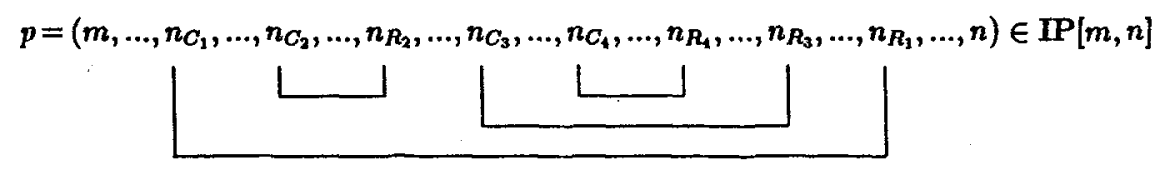

Figure 3: Complete Interprocedural Paths

This pattern is illustrated in Figure 3 , where $\left\{\left(n_{C_{i}}, n_{R_{i}}\right) \mid i \in\{1, \ldots, 4\}\right\}$ are assumed to be pairs of corresponding call and return nodes of $p$.

The following lemma, which can easily be proved, will be important:

Lemma 3.5 Let $\mathrm{s} \in\left\{\mathrm{s}_{0}, \ldots, \mathrm{s}_{k}\right\}, p=\left(n_{1}, \ldots, n_{k}\right) \in \operatorname{IP}[\mathrm{s}, n]$ and $\left(n_{i}, n_{j}\right)$ a pair of corresponding call and return nodes. Then we have:

$$
\left(n_{i+1}, \ldots, n_{j-1}\right) \in \operatorname{CIP}\left[\operatorname{start}\left(\text { callee }\left(n_{i}\right)\right), \text { end }\left(\text { callee }\left(n_{i}\right)\right)\right]
$$

Remark 3.6 If the underlying program $\Pi$ has no procedures, the flow graph system $S$ and the interprocedural flow graph $G^{*}$ collapse to the flow graph $G_{0}$ of $\pi_{0}$. In this special case our framework coincides with the standard intraprocedural framework.

Conventions: Throughout the rest of this paper we assume an arbitrary but fixed program $\Pi=\left(\pi_{0}, \pi_{1}, ., \pi_{k}\right)$ with flow graph system $S=\left(G_{0}, G_{1}, ., G_{k}\right)$ and interprocedural flow graph $G^{*}=$ $\left(N^{*}, E^{*}, \mathrm{~s}^{*}, \mathrm{e}^{*}\right)$. Moreover, $m$ and $n$, possibly indexed, are nodes of $S$ or $G^{*}$, and for every node $n \in \mathrm{N}_{C}^{S}, n_{C}$ and $n_{R}$ denote its corresponding call node and return node in $N^{*}$, respectively.

\section{Abstract Semantics}

In this section we present new interprocedural versions of the meet over all paths strategy and the maximal fixed point strategy. They define the (global) semantics for interprocedural flow graphs and flow graph systems, respectively. The point of this presentation is the extension of the data flow information in a way that mimics run-time stacks as used in run-time systems.

\subsection{The Local Semantic Functional}

As its intraprocedural counterpart, the interprocedural meet over all paths (IMOP) solution directly records all possible program executions that, lead to a particular program point. However, in the presence of recursive procedures it is necessary to work on stacks of lattice elements instead of the lattice itself, in order to record the part of the history which will become relevant after returning from (nested) procedure calls. Thus the local semantic functional has type

$$
\text { [ ] : } N^{*} \rightarrow(S T A C K \rightarrow S T A C K)
$$

where STACK denotes the set of all non empty stacks with components of $\mathcal{C}$, which can be manipulated by means of the following operations:

- newstack : $\mathcal{C} \rightarrow S T A C K$

- push : STACK $\times \mathcal{C} \rightarrow S T A C K$

- pop : STACK $\rightarrow$ STACK

- top : STACK $\rightarrow \mathcal{C}$ 
Intuitively, newstack(c) creates a new stack with single component $c$, push puts a new component on the top of the argument stack, pop removes the top component, and top delivers the content of the top component, while not affecting the argument stack. Thus only the top components of the stacks can be affected by these operations.

STACK is an abstract version of the run-time stacks used by run-time systems for maintaining the activation records of different procedure incarnations. Intuitively, the top component of a stack contains the data flow information corresponding to the currently valjd activation record ${ }^{8}$, while the data flow informations of the remaining stack components correspond to activation records of preceding but not yet finished procedure calls. However, in contrast to a concrete run-time stack, where variables that are global for the currently activated procedure are accessed by means of static and dynamic link chains, the components of a data flow analysis stack are assumed to contain all information related with the current procedure incarnation, i.e. also the information related to global variables ${ }^{9}$. Thus the data flow analysis stacks directly reflect the nesting of procedure incarnations according to the current call sequence.

Formally, the local semantic functional $\llbracket *$ for this setting is defined by

$\forall n \in N^{*} \forall s t k \in S T A C K$.

$$
\llbracket n \rrbracket^{*}(s t k)=d_{d f}\left\{\begin{array}{lc}
\operatorname{push}\left(\operatorname{pop}(s t k), \llbracket n \rrbracket^{\prime}(t o p(s t k))\right) & \text { if } n \in N^{*} \backslash\left(N_{C}^{*} \cup N_{R}^{*}\right) \\
\operatorname{push}\left(\operatorname{stk}, \llbracket n \rrbracket^{\prime}(\operatorname{top}(\operatorname{stk}))\right) & \text { if } n \in N_{C}^{*} \\
\operatorname{push}\left(\operatorname{pop}(\operatorname{pop}(\operatorname{stk})), \mathcal{R}_{n}\left(\operatorname{top}(\operatorname{pop}(s t k)), \llbracket n \rrbracket^{\prime}(\operatorname{top}(s t k))\right)\right) & \text { if } n \in N_{R}^{*}
\end{array}\right.
$$

where $\llbracket: N^{*} \rightarrow(\mathcal{C} \rightarrow \mathcal{C})$ denotes the straightforward extension of the semantic functional of Section 2 to interprocedural flow graphs ${ }^{10}$, and $\mathcal{R}_{n}: \mathcal{C} \times \mathcal{C} \rightarrow \mathcal{C}$ is a function as described below.

The intuition behind this definition is as follows:

The execution of an ordinary statement (i.e. $n \in N^{*} \backslash\left(N_{C}^{*} \cup N_{R}^{*}\right)$ ) only affects the currently valid activation record. Thus it can be modelled by simply modifying the top component of the stack representing the current data flow information.

A procedure call (i.e. $n \in N_{C}^{*}$ ) requires the generation of a new activation record. This is reflected by pushing a new element on the top of the stack, which results from modifying the top component of the stack according to the parameter transfer.

The treatment of return statements (i.e. $n \in N_{R}^{*}$ ) demonstrates the necessity of introducing stacks into the framework. Returning from a procedure call (i.e. $n \in N_{R}^{*}$ ) essentially requires removal of the activation record belonging to the called procedure and reactivation of its predecessor. However, one observation is important here. The effect of a (directly) recursive procedure to a global variable needs to be maintained, whereas the local variables must be reset to their values at call time. Thus we need to consider the data flow information valid immediately before entering the procedure (available in top $(p o p(s t k))$ ), as well as the information valid after executing its body (available in $\left.\llbracket n \rrbracket^{\prime}(\operatorname{top}(s t k))\right)$, in order to compute the data flow information being valid after returning from the called procedure. The function $\mathcal{R}_{n}: \mathcal{C} \times \mathcal{C} \rightarrow \mathcal{C}$ models this computation. Thus popping the top component of the stack and replacing the subsequent component by

$$
\mathcal{R}_{n}\left(\operatorname{top}(\operatorname{pop}(s t k)), \llbracket n \mathbb{1}^{\prime}(t o p(s t k))\right)
$$

reflects the whole process of completing a procedure call.

\footnotetext{
${ }^{7}$ We consider the operation nerostack instead of the usual emptystack : $\rightarrow$ STACK here, in order to exclude empty stacks, which are irrelevant in our framework.

${ }^{8}$ Therefore, we are never dealing with empty stacks.

${ }^{9}$ Static and dynamic link chains are just a technical mean for getting efficient implementations of run-time systems. In our abstract framework, however, this aspect can be neglected withont any harm (cf. Remark 4.7). Moreover, it allows us to work with local semantic functionals that affect only the top components of data flow analysis stacks.

${ }^{10}[n]^{*}(s t k), n \in N_{R}^{*}$, is only defined for stacks with at. least, two components, a fact, which is automatically taken care of in any reasonable analysis context.
} 


\subsection{The Structure of the Semantic Functions}

Let $\mathcal{F}={ }_{d j}[S T A C K \rightarrow S T A C K]$ denote the set of all functions from STACK to' STACK and

$$
\begin{aligned}
& \mathcal{F}_{O}={ }_{d f}\{f \in \mathcal{F} \mid \forall s t k \in S T A C K . \operatorname{pop}(f(s t k))=\operatorname{pop}(s t k)\} \\
& \mathcal{F}_{C}=_{d f}\{f \in \mathcal{F} \mid \forall s t k \in S T A C K . \operatorname{pop}(f(s t k))=s t k\} \\
& \mathcal{F}_{R}={ }_{d f}\{f \in \mathcal{F} \mid \forall s t k \in S T A C K . \operatorname{pop}(f(s t k))=\operatorname{pop}(\operatorname{pop}(s t k))\}
\end{aligned}
$$

Then we have:

\section{Lemma 4.1}

$$
\begin{aligned}
& \text { 1. } \forall n \in N^{*} \backslash\left(N_{C}^{*} \cup N_{R}^{*}\right) \cdot \llbracket n \rrbracket^{*} \in \mathcal{F}_{O} \\
& \text { 2. } \forall n \in N_{C}^{*} \cdot \llbracket n \rrbracket^{*} \in \mathcal{F}_{C} \\
& \text { 3. } \forall n \in N_{R}^{*} \cdot \llbracket n \rrbracket^{*} \in \mathcal{F}_{R}
\end{aligned}
$$

Intuitively this means that the semantic function of an ordinary statement only affects the top component of the argument stack, that the semantic function of a call statement simply adds a new top component to the argument stack, and that a return statement replaces the upper two components of the argument stack by a new component. The following lemma is an easy consequence of these properties of $\mathcal{F}_{O}, \mathcal{F}_{C}$ and $\mathcal{F}_{R}$.

Lemma 4.2 $\forall f_{r} \in \mathcal{F}_{R} \quad \forall f_{o}, f_{o}^{\prime} \in \mathcal{F}_{O} \quad \forall f_{c} \in \mathcal{F}_{C} . \quad f_{o} \circ f_{o}^{\prime}, f_{r} \circ f_{o} \circ f_{c} \in \mathcal{F}_{O}$

The formal development of the paper requires the following derived notions of monotonicity and distributivity:

\section{Definition 4.3 (S-Monotonicity, S-Distributivity)}

A function $f \in \mathcal{F}_{O} \cup \mathcal{F}_{C} \cup \mathcal{F}_{R}$ is called

- s-monotonic iff $f_{s}$ is monotonic

- s-distributive iff $f_{s}$ is distributive

where $f_{s}$, the significant part of $f$, is defined according to the following two cases:

- $f \in \mathcal{F}_{o} \cup \mathcal{F}_{C}:$ here $f_{s}: \mathcal{C} \rightarrow \mathcal{C}$ is defined by: $f_{s}(c)={ }_{d f}$ top $(f($ newstack $(c)))$

- $f \in \mathcal{F}_{R}:$ here $f_{s}: \mathcal{C} \times \mathcal{C} \rightarrow \mathcal{C}$ is defined by ${ }^{11}: f_{s}\left(c_{1}, c_{2}\right)={ }_{d f} \operatorname{top}\left(f\left(p u s h\left(\right.\right.\right.$ newstack $\left.\left.\left.\left(c_{1}\right), c_{2}\right)\right)\right)$

The following lemma shows that the effort for checking the preconditions of the Interprocedural Safety Theorem $\mathbf{5 . 2}$ and the Interprocedural Coincidence Theorem 5.3 is comparable to the effort necessary for their intraprocedural counterparts (cf. Section 2 and 5 ).

Lemma 4.4 For all $n \in N^{*}$ we have that $\llbracket n \rrbracket^{*}$ is s-monotonic (s-distributive) if

$\left.n \in N_{R}^{*}: \llbracket n\right\rceil^{\prime}$ and $\mathcal{R}_{n}$ are monotonic (distributive)

$n \notin N_{R}^{*}:\lfloor n]^{\prime}$ is monotonic (distributive)

Conventions: In the following we consider s-monotonicity (s-distributivity) as a generalization of the usual monotonicity (distributivity) by identifying lattice elements with their unique representations as one-component stacks. Moreover, we extend the meet operation $\Pi$ to work on stacks in the following way:

$$
\forall S T K \subseteq S T A C K . \Pi S T K==_{d f} \text { newstack }\left(\prod\{t o p(s t k) \mid s t k \in S T K\}\right)
$$

Thus, the meet over a set of stacks is just the one-component stack containing the meet of all the top components in its single component.

\footnotetext{
${ }^{11}$ Note that $\mathcal{C} \times \mathcal{C}$ is a lattice, whenever $\mathcal{C}$ is.
} 


\subsection{The Interprocedural Meet Over all Paths Solution}

Analogously to Section 2 the local abstract semantics []$^{*}$ can be extended to cover finite interprocedural paths. For every path $p=\left(n_{1}, \ldots, n_{q}\right) \in \mathbb{I P}[m, n]$, we define $\llbracket p \rrbracket^{*}:$ STACK $\rightarrow$ STACK by

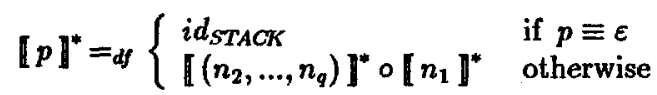

Now, as its intraprocedural counterpart (cf. [KU]), the interprocedural meet over all paths (IMOP) solution directly records all possible program executions leading to a particular program point. Here it is important that for any interprocedural path $p \in \operatorname{IP}\left[\mathbf{s}^{*}, n\right)$ and any stack stk $\in S T A C K$, $\left.\operatorname{top}(\mathbb{[} p]^{*}(s t k)\right)$ is the only data flow information relevant for node $n$ after executing $p$, since all other components of $\llbracket p \rrbracket^{*}(s t k)$ correspond to activation records that are not valid after $p$. Identifying one-component stacks with the content of their unique component, the formal definition of the interprocedural meet over all paths solution is given by:

The IMOP-Solution: $\forall n \in N^{*} \forall c_{0} \in \mathcal{C} . J M O P_{c_{0}}(n)=\prod\left\{\llbracket p \rrbracket^{*}\left(n e w s t a c k\left(c_{0}\right)\right) \mid p \in \mathbb{I P}\left[\mathbf{s}^{*}, n\right)\right\}$

\subsection{The Interprocedural Maximal Fixed Point Solution}

In addition to the equational characterization of the intraprocedural case (Equation System 2.1), flow graph systems need a preprocess, which determines the meaning of call nodes in terms of the meaning of the called procedures. This requires the introduction of an auxiliary semantic functional 【I, which gives meaning to whole flow graphs. Essentially, $n \mathbb{n}$ transforms data flow information that is assumed to be valid at the entry of the procedure that contains $n$ into the corresponding data flow information being valid before an execution of $n$. In particular, If $e_{i}$ is the meaning function of the $\mathrm{i}$-th procedure ${ }^{12}$. Formally, the full preprocess for determining the meaning $\llbracket n \rrbracket$ of call nodes $n \in \mathrm{N}_{C}^{S}$ is characterized by:

Definition 4.5 \: $\mathrm{N}^{S} \rightarrow(S T A C K \rightarrow S T A C K)$ and \\: $\mathrm{N}^{S} \rightarrow(S T A C K \rightarrow S T A C K)$ are defined as the greatest solution of the equation system given by:

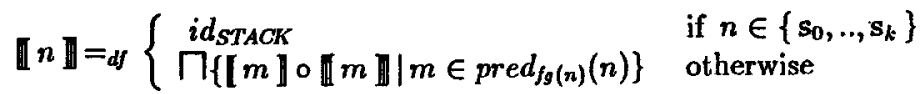

and

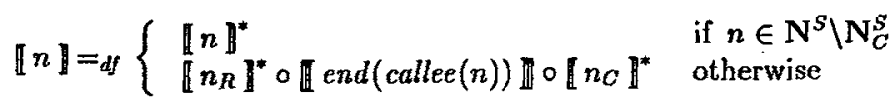

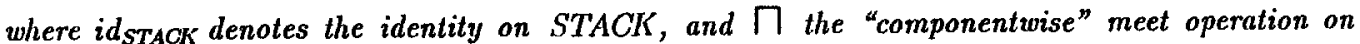
$\mathcal{F}_{0}{ }^{13}$

The effect of a procedure call $n \in \mathbf{N}_{C}^{S}$ is determined in three steps reflecting the three phases of its execution:

- Entering the called procedure: $\llbracket n_{C} \rrbracket^{*}$ creates a new activation record by transforming the content of the top component of the stack according to the semantics of the call node and pushing it onto the stack. - Usually, the semantics of call nodes will reflect the parameter transfer.

\footnotetext{
${ }^{12}$ Remember, $\left[e_{i}\right]^{\prime}=d f i d c$. Thus, $e_{i}$ is related to the identity on $S T A C K$.

${ }_{13} \forall f, f^{\prime} \in \mathcal{F}_{O} . f \Pi f^{\prime}=d f f^{\prime \prime} \in \mathcal{F}_{O}$ with $\forall$ stk $\in \operatorname{STACK} . \operatorname{top}\left(f^{\prime \prime}(\operatorname{st} k)\right)=\operatorname{top}(f(s t k)) \cap \operatorname{top}\left(f^{\prime}(s t k)\right)$. As usual, "ח" induces an inclusion relation "ㄷ" on $\mathcal{F}_{0}$ by: $f \subseteq f^{\prime}$ iff $f \cap f^{\prime}=f$.
} 


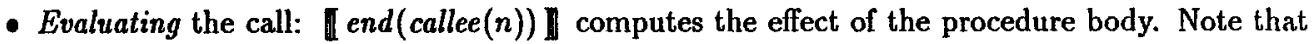
this affects the top component of the argument stack only.

- Leaving the called procedure: $\left.\llbracket n_{R}\right\rceil^{*}$ removes the activation record related with the current procedure call by popping the top component from the stack, and replacing its subsequent component by the data flow information representing the effect of the procedure call relative to its call site.

Applying Lemma 4.2, we obtain

Lemma 4.6 $\forall n \in \mathrm{N}^{S}$. $n \llbracket, \llbracket n \rrbracket \in \mathcal{F}_{O}$

Remark 4.7 Lemma 4.6 is important, since it shows that all the stacks occurring during the iterative computation of the IMFP-solution will have at most two components ${ }^{14}$. This is in contrast to the IMOP-strategy, where the size of stacks contributing to the $I M O P$-solution is in general unbounded. Moreover, it allows us to prove termination in the usual way.

After fixing the meaning of call nodes, 【 I plays essentially the same role as the local (abstract) semantic functional of Section 2. Formally, the interprocedural maximal fixed point strategy is characterized by Equation System 4.8. As its intraprocedural counterpart, this strategy labels every node $n$ of $\mathrm{N}^{S}$ with a pre-information pre $c_{c_{0}}(n)$ and a post-information post $c_{c_{0}}(n)$, whose top components are the greatest solution of this equation system with respect to $c_{0} \in \mathcal{C}$.

\section{Equation System 4.8}

$$
\begin{aligned}
& \operatorname{pre}(n)= \begin{cases}n e w s t a c k\left(c_{0}\right) & \text { if } n=\mathbf{s}_{0} \\
\prod_{\prod}\left\{m_{C} \rrbracket^{*}(\operatorname{pre}(m)) \mid m \in \operatorname{caller}(f g(n))\right\} & \text { if } n \in\left\{\mathbf{s}_{1}, ., \mathbf{s}_{k}\right\} \\
\prod_{\left\{\operatorname{post}(m) \mid m \in \operatorname{pred}_{f_{g}(n)}(n)\right\}} & \text { otherwise }\end{cases} \\
& \operatorname{post}(n)=\llbracket n \rrbracket(\operatorname{pre}(n))
\end{aligned}
$$

As before, identifying a stack having a single component only with the content of this component, we obtain as in the intraprocedural case:

The IMFP-Solution: $\forall n \in N^{S} \forall c_{0} \in \mathcal{C} . I M F P_{c_{0}}(n)=\operatorname{pre}_{c_{0}}(n)$

\section{Main Results}

The main step in the proof of our main results is taken by proving the following Main Lemma 5.1, whose proof is given in full detail in [KS1].

Lemma 5.1 (The Main Lemma)

For all $n \in \mathbf{N}_{C}^{S}$, we have, if the semantic functions $\llbracket m \rrbracket^{*}, m \in N^{*}$, are

1. s-monotonic: $\llbracket n \rrbracket \sqsubseteq \prod\left\{\left[p \rrbracket^{*} \mid p \in \operatorname{CIP}\left[n_{C}, n_{R}\right]\right\}\right.$

2. s-distributive: $\llbracket n\rceil=\prod\left\{\left\lfloor p \rrbracket^{*} \mid p \in \operatorname{CIP}\left[n_{C}, n_{R}\right]\right\}\right.$

\footnotetext{
${ }^{14}$ This is because the computation starts from a one-component stack newstack( $\left.c_{0}\right)$ (cf. Equation System 4.8 and Algorithm A.3).
} 
After having established this result, the Interprocedural Safety Theorem 5.2 and the Interprocedural Coincidence Theorem 5.3 can be proved almost as in the intraprocedural case. Thus we omit these proofs here ${ }^{15}$.

As in the intraprocedural case, the first theorem states that the IMFP-solution is a correct approximation of the $I M O P$-solution, whenever all the local abstract semantic functions are s-monotonic:

\section{Theorem 5.2 (Interprocedural Safety Theorem)}

Given a flow graph system $S=\left(G_{0}, G_{1}, ., G_{k}\right)$ and its corresponding interprocedural flow graph $G^{*}=\left(N^{*}, E^{*}, \mathrm{~s}^{*}, \mathrm{e}^{*}\right)$, the IMFP-solution is a correct approximation of the IMOP-solution, i.e. $\forall n \in \mathbb{N}^{S} \forall c_{0} \in \mathcal{C}$. IMFP $c_{c_{0}}(n) \subseteq I M O P_{c_{0}}(n)$, if the abstract semantics 【n $\prod^{*}$ of all nodes $n \in N^{*}$ is given by an s-monotonic function.

Again, as in the intraprocedural case, s-distributivity of the semantic functions yields optimality (or completeness):

\section{Theorem 5.3 (Interprocedural Coincidence Theorem)}

Given a flow graph system $S=\left(G_{0}, G_{1}, ., G_{k}\right)$ and its corresponding interprocedural flow graph $G^{*}=\left(N^{*}, E^{*}, \mathrm{~s}^{*}, \mathrm{e}^{*}\right)$, the IMFP-solution and the IMOP-solution coincide, i.e. $\forall n \in \mathrm{N}^{S} \forall c_{0} \in$ C. IMOP $c_{c_{0}}(n)=I M F P_{c_{0}}(n)$, if the abstract semantics $\llbracket n \rrbracket^{*}$ of all nodes $n \in N^{*}$ is given by an s-distributive function.

Note that Lemma 4.4 allows to check the s-monotonicity or s-distributivity of the semantic functions $\llbracket n \rrbracket^{*}$ simply by checking these properties for the semantic functions $\llbracket n \rrbracket^{\prime}$ and the reduction functions $\mathcal{R}_{n}$. Thus the only additional effort in comparison to the intraprocedural case arises from checking the reduction functions.

\section{Applications}

In this section we sketch two applications of the Interprocedural Coincidence Theorem 5.3. We omit details here, since both examples require their own setup.

In [SK2] we propose an algorithm for interprocedural constant propagation and constant folding, which generalizes and improves all previous techniques for interprocedural constant propagation (cf. [CC2, CCKT, JM]). This algorithm determines all finite interprocedural constants, which are the interprocedural analogue to the set of finite constants introduced in [SK1]. As in the intraprocedural case, finite interprocedural constants have a purely operational characterization in the sense of the $I M O P$-strategy, and a purely denotational characterization in the sense of the $I M F P$-strategy. The Interprocedural Coincidence Theorem 5.3 yields the equivalence of these characterizations.

The second example concerns the interprocedural versions of the classical bit-vector data flow analyses, e.g. determining available expressions, reaching definitions, live variables, very busy (anticipatable) expressions (cf. [He]) ${ }^{16}$, and, more sophisticatedly, the optimal elimination of interprocedural partial redundancies ${ }^{17}$. In all these cases, the Interprocedural Coincidence Theorem 5.3 allows us to prove the optimality of our algorithms for programs with recursive procedures, global and local variables, and formal value parameters $[\mathrm{KS} 2]^{18}$.

\footnotetext{
${ }^{15}$ Both proofs are given in [KS1].

${ }^{16}$ This application has also been suggested (without giving any details) by Sharir and Pnueli [SP].

${ }^{17}$ This problem is heuristically dealt with in [Mo, MR].

${ }^{18} \mathrm{~A}$ detailed presentation of interprocedural bit-vector data flow analyses is given in [KS3].
} 


\section{Conclusions}

We have presented an interprocedural generalization of the well-known intraprocedural Coincidence Theorem of Kam and Ullman [KU], which covers arbitrary programs with recursive procedures, global and local variables, and formal value parameters. Our theorem, which reduces to the classical intraprocedural version in the absence of procedures, delivers a sufficient condition for the coincidence of the interprocedural meet over all paths strategy and the interprocedural maximal fixed point strategy, and it generalizes previous results (cf. [Ba1, Ba2, SP]), which do not deal properly with local variables of recursive procedures. Our results are formulated within the framework of abstract interpretation, thus covering a wide range of data flow analyses.

\section{References}

[All] Allen, F. E. Control flow analysis. SIGPLAN Not. 5, 7 (1970), 1 - 19.

[Ba1] Barth, G. Interprozedurale Datenflußsysteme. Habilitationsschrift, University of Kaiserslautern, Germany, 1981.

[Ba2] Barth, G. Interprocedural data flow systems. In Proceedings $6^{\text {th }}$ GI-Conference, Dortmund, Germany, Springer-Verlag, LNCS 145 (1983), 49 - 59.

[Bo] Bourdoncle, F. Interprocedural abstract interpretation of block structured languages with nested procedures, aliasing and recursivity. In Proceedings $2^{\text {nd }}$ PLILP, Linköping, Sweden, Springer-Verlag, LNCS 456 (1990), 307 - 323.

[CC1] Cousot, P., and Cousot, R. Abstract interpretation: A unified lattice model for static analysis of programs by construction or approximation of fixpoints. In Proceedings $4^{\text {th }}$ POPL, Los Angeles, California, 1977, 238 - 252.

[CC2] Cousot, P., and Cousot, R. Static determination of dynamic properties of recursive procedures. In: Neuhold, E. (Ed.). Proceedings of the $2^{\text {nd }}$ IFIP TC-2 Working Conference on Formal Description of Programming Concepts, St. Andrews, N. B., Canada, 1977, 237 . 277.

[CCKT] Callahan, D., Cooper, K. D., Kennedy, K. W., and Torczon, L. M. Interprocedural constant propagation. In Proceedings SIGPLAN'86 Symp. on Compiler Construction, SIGPLAN Not. 21, 7 (1986), 152 - 161.

[He] Hecht, M. S. Flow analysis of computer programs. Elsevier, North-Holland, 1977.

[JM] Jones, N. D., and Muchnick, S. S. A flexible approach to interprocedural data flow analysis and programs with recursive data structures. In Proceedings $9^{\text {th }}$ POPL, Albuquerque, New Mexico, 1982, 66 - 74.

[Ki] Kildall, G. A. A unified approach to global program optimization. In Proceedings $1^{\text {st }}$ POPL, Boston, Massachusetts, 1973, $194-206$.

[KS1] Knoop, J., and Steffen, B. The interprocedural coincidence theorem. Aachener InformatikBerichte Nr. 91-27, Rheinisch-Westfälische Technische Hochschule Aachen, Aachen, Germany, 1991.

[KS2] Knoop, J., and Steffen, B. Optimal interprocedural partial redundancy elimination. In $A d-$ denda to Proceedings $f^{\text {th }} C C$, Paderborn, Germany, October 5-7, 1992. Technical Report, Department of Computer Science, University of Paderborn, Germany, 1992. 
[KS3] Knoop, J., and Steffen, B. Efficient and optimal bit-vector data flow analyses: A uniform interprocedural framework. To appear.

[KU] Kam, J. B., and Ullman, J. D. Monotone data flow analysis frameworks. Acta Informatica 7, (1977), 309 - 317.

[La] Langmaack, H. On procedures as open subroutines. Part I. Acta Informatica 2, (1973), $311-333$.

[Mo] Morel, E. Data flow analysis and global optimization. In: Lorho, B. (Ed.). Methods and tools for compiler construction. Cambridge University Press, 1984, 289 - 315.

[My] Myers, E. W. A precise inter-procedural data flow algorithm. In Proceedings $8^{\text {th }} P O P L$, Williamsburg, Virginia, 1981, 219 - 230.

[MJ] Muchnick, S. S., and Jones, N. D. (Eds.). Program flow analysis: Theory and applications. Prentice Hall, Englewood Cliffs, New Jersey, 1981.

[MR] Morel, E., and Renvoise, C. Interprocedural elimination of partial redundancies. In [MJ], $1981,160-188$.

[Ro] Rosen, B. K. Data flow analysis for procedural languages. Journal of the ACM 26, 2 (1979), $322-344$.

[SK1] Steffen, B., and Knoop, J. Finite constants: Characterizations of a new decidable set of constants. In Proceedings $14^{\text {th }}$ MFCS, Pora̧bka-Kozubnik, Poland, Springer-Verlag, LNCS 379 (1989), 481 - 491. An extended version appeared in: Theoretical Computer Science 80, 2 (1991), $303-318$.

[SK2] Steffen, B., and Knoop, J. Finite interprocedural constants. To appear.

[SP] Sharir, M., and Pnueli, A. Two approaches to interprocedural data flow analysis. In [MJ], 1981, 189 - 233.

\section{A Algorithms}

This section provides algorithms that compute the IMFP-solution. First, we have an algorithm for the preprocess that determines the semantics of call nodes:

\section{Algorithm A.1 (Computation of the Semantic Functionals I I and II}

Input: A flow graph system $S=\left(G_{0}, G_{1}, ., G_{k}\right)$, a complete semi-lattice $\mathcal{C}$, for every node $n \in$ $\mathrm{N}^{S} \backslash \mathrm{N}_{C}^{S}$ an s-monotonic function $\left[n \mathbb{1}^{*}: S T A C K \rightarrow S T A C K \in \mathcal{F}_{O}\right.$, which is the identity for all nodes in $\left\{\mathrm{s}_{0}, \ldots, \mathrm{s}_{k}, \mathrm{e}_{0}, \ldots, \mathbf{e}_{k}\right\}$. Moreover, for every node $n \in \mathbf{N}_{C}^{S}$ two s-monotonic functions $\llbracket n_{C} \rrbracket^{*}$ : $S T A C K \rightarrow S T A C K \in \mathcal{F}_{C}$ and $\llbracket n_{R} \rrbracket^{*}: S T A C K \rightarrow S T A C K \in \mathcal{F}_{R}$. All semantic functions $\llbracket n \rrbracket^{*}$, $n \in N^{*} \backslash N_{R}^{*}$, are assumed to map stacks with top component $\mathrm{T}$ to stacks with top component $\mathrm{T}$. Analogously, all semantic functions $[n]^{*}, n \in N_{R}^{*}$, are assumed to map stacks with upper two components $T$ to stacks with top component $T$.

Output: An annotation of $S$ with functions $n n: S T A C K \rightarrow S T A C K$ (stored in gtr) and 【n $n:$ STACK $\rightarrow$ STACK (stored in ltr) that satisfy Definition 4.5.

Remark: $\mathrm{T}_{\mathcal{F}_{o}}: S T A C K \rightarrow S T A C K \in \mathcal{F}_{\mathcal{O}}$ denotes the "universal" function which is assumed to "contain" every function $f \in \mathcal{F}_{O}$, and id STACK $_{\text {is }}$ is the identity on STACK. The variable workset controls the iterative process. Its elements are tuples, whose first components are nodes $m \in \mathbf{N}^{S}$ of the flow graph system $S$, and whose second components are functions $f: S T A C K \rightarrow S T A C K \in \mathcal{F}_{0}$ 
that specify a new approximation for the function $m$ of the node of the first component. Note that due to the mutual interdependence of the definitions of 1 and [ the iterative approximation of II is superposed by an interprocedural iteration step which updates the semantics [1 of call nodes.

(Initialization of the annotation arrays gtr and ltr and the variable workset)

FORALL $m \in \mathbf{N}^{s}$ DO

$\operatorname{gtr}[m]:=\mathrm{T}_{\mathcal{F}_{0}}$;

IF $m \in \mathbf{N}_{C}^{S}$ THEN $l t r[m]:=T_{\mathcal{F}_{0}}$ ELSE $l t r[m]:=[m]^{*}$ FI

OD;

workset $:=\left\{\left(\mathbf{s}, i d_{S T A C K}\right) \mid s \in\left\{s_{0}, \ldots, s_{k}\right\}\right\}$

(Iterative fixed point computation)

WHILE workset $\neq 0$ DO

LET $(m, f) \in$ workset

BEGIN

workset $:=$ workset $\backslash\{(m, f)\}$

IF $\operatorname{gtr}[m] \sqsupset \operatorname{gtr}[m] \sqcap f$

THEN

$\operatorname{gtr}[m]:=\operatorname{gtr}[m] \sqcap f$

IF $m \in\left\{\mathbf{e}_{i} \mid i \in\{0, ., k\}\right\}$

THEN

FORALL $l \in$ caller $(f g(m))$ DO

$\operatorname{ltr}[l]:=\llbracket l_{R} \rrbracket^{*} \circ \operatorname{gtr}[m] \circ \llbracket l_{C} \rrbracket^{*} ;$

OD

workset $:=$ workset $\cup\left\{(n, l \operatorname{lt}[l] \circ g \operatorname{tr}[l]) \mid n \in \operatorname{succ}_{g(l)}(l)\right\}$

ELSE

FI

workset $:=$ workset $\cup\left\{(n, \operatorname{ltr}[m] \circ \operatorname{gtr}[m]) \mid n \in \operatorname{succ}_{g_{g(m}(m)}(m)\right\}$

FI

END

OD.

In order to simplify the formulation of the central property of this algorithm, we abbreviate the values of $l t r[n]$ and $g t r[n]$ after the $k$-th execution of the while-loop by $l t r^{k}[n]$ and $g t r^{k}[n]$, respectively. The following theorem can now be proved in a straightforward fashion (cf. [Ki]):

Theorem A.2 $\left.\forall n \in \mathbb{N}^{S} . \llbracket n\right]=\prod\left\{l r^{k}[n] \mid k \geq 0\right\} \quad \wedge \quad \| n \rrbracket=\Pi\left\{g t r^{k}[n] \mid k \geq 0\right\}$

In particular, we have $\forall n \in \mathbf{N}^{s}$. $n \llbracket=\operatorname{gtr}[n] \wedge \llbracket n \rrbracket=l \ln [n]$ after termination of Algorithm A.1.

The second algorithm computes the IMFP-Solution:

\section{Algorithm A.3 (Computation of the IMFP-Solution)}

Input: A flow graph system $S=\left(G_{0}, G_{1}, ., G_{k}\right)$, the semantic functional $\llbracket$, for every node $n \in \mathbb{N}_{C}^{S}$ the function $\llbracket n_{C} \rrbracket^{*}$, and a start information $c_{0} \in \mathcal{C}$. All semantic functions $\llbracket n \rrbracket$ and $\llbracket n_{C} \rrbracket^{*}$ are assumed to be s-monotonic and to map stacks with top component $T$ to stacks with top component $\mathrm{T}$.

Output: An annotation of $S$ with data flow informations, i.e. an annotation with pre-informations (stored in pre) and post-informations (stored in post) of one-component stacks that characterize valid data flow information at the entry and at the exit of every node. 
Remark: newstack $(T)$ denotes the "universal" data flow information, which is assumed to "contain" every data flow information. The variable workset controls the iterative process. Its elements are tuples whose first components are nodes $m \in \mathbf{N}^{S}$ of the flow graph system $S$ and whose second components are elements of STACK specifying a new approximation for the pre-information of the node of the first component.

(Initialization of the annotation arrays pre and post and the variable workset)

FORALL $m \in \mathbb{N}^{S}$ DO $($ pre $[m]$, post $[m]):=($ newstack $(T)$, newstack $(\mathrm{T}))$ OD;

workset $:=\left\{\left(\mathrm{s}_{0}\right.\right.$, newstack $\left.\left.\left(c_{0}\right)\right)\right\}$;

(Iterative fixed point computation)

WHILE workset $\neq \emptyset$ DO

LET $(m, s t k) \in$ workset

BEGIN

workset $:=$ workset $\backslash\{(m, s t k)\} ;$

IF pre $[m] \sqsupset \operatorname{pre}[m] \sqcap s t k$

THEN

$\operatorname{pre}[m]:=\operatorname{pre}[m] \sqcap s t k$

post $[m]:=\llbracket m \rrbracket($ pre $[m])$;

workset $:=$ workset $\cup\left\{(n, \operatorname{post}[m]) \mid n \in \operatorname{succ}_{J_{g}(m)}(m)\right\}$;

IF $m \in \mathbf{N}_{C}^{S}$

THEN workset $:=\operatorname{workset} \cup\left\{\left(\operatorname{start}(\operatorname{callee}(m)), \llbracket m_{C} \rrbracket^{*}(\right.\right.$ pre $\left.\left.[m])\right)\right\}$

FI FI

END

OD.

As before, given a start information $c_{0}$, we abbreviate the values of pre[n], and post $[n]$ after the $k$-th execution of the while-loop by $p r e^{k}[n]$, and $\operatorname{post}^{k}[n]$. In analogy to Theorem A.2 we have:

Theorem A.4 $\left.\forall n \in \mathbb{N}^{S} . \operatorname{IMFP}_{c_{0}}(n)=\prod_{\{p r e}[n] \mid k \geq 0\right\}$

In particular, we have $\forall n \in \mathrm{N}^{S} . \operatorname{IMFP}_{\mathrm{c}_{0}}(n)=$ pre[n] after termination of Algorithm A.3. 